\title{
RESEARCH
}

\section{Dosimetry of 3 CBCT devices for oral and maxillofacial radiology: CB Mercuray, NewTom 3G and i-CAT}

\author{
JB Ludlow*,1, LE Davies-Ludlow ${ }^{2}$, SL Brooks $^{3}$ and WB Howerton ${ }^{4}$ \\ ${ }^{1}$ Department of Diagnostic Sciences and General Dentistry, University of North Carolina School of Dentistry, Chapel Hill, North \\ Carolina, USA; ${ }^{2}$ University of North Carolina School of Dentistry, Chapel Hill, North Carolina, USA; ${ }^{3}$ Department of Periodontics and \\ Oral Medicine, University of Michigan School of Dentistry, Ann Arbor, Michigan, USA; ${ }^{4}$ Private practice of Oral and Maxillofacial \\ Radiology, Raleigh, NC, USA
}

\begin{abstract}
Objectives: Cone beam computed tomography (CBCT), which provides a lower dose, lower cost alternative to conventional $\mathrm{CT}$, is being used with increasing frequency in the practice of oral and maxillofacial radiology. This study provides comparative measurements of effective dose for three commercially available, large (12") field-of-view (FOV), CBCT units: CB Mercuray, NewTom 3G and i-CAT.

Methods: Thermoluminescent dosemeters (TLDs) were placed at 24 sites throughout the layers of the head and neck of a tissue-equivalent human skull RANDO phantom. Depending on availability, the $12^{\prime \prime}$ FOV and smaller FOV scanning modes were used with similar phantom positioning geometry for each CBCT unit. Radiation weighted doses to individual organs were summed using $1990\left(E_{1990}\right)$ and proposed $2005\left(E_{2005 \text { draft }}\right)$ ICRP tissue weighting factors to calculate two measures of whole-body effective dose. Dose as a multiple of a representative panoramic radiography dose was also calculated.

Results: For repeated runs dosimetry was generally reproducible within $2.5 \%$. Calculated doses in $\mathrm{mSv}\left(E_{1990}, E_{2005 \text { draft }}\right)$ were NewTom3G $(45,59)$, i-CAT $(135,193)$ and CB Mercuray $(477,558)$. These are 4 to 42 times greater than comparable panoramic examination doses $(6.3 \mathrm{mSv}, 13.3 \mathrm{mSv})$. Reductions in dose were seen with reduction in field size and $\mathrm{mA}$ and $\mathrm{kV}$ technique factors.

Conclusions: CBCT dose varies substantially depending on the device, FOV and selected technique factors. Effective dose detriment is several to many times higher than conventional panoramic imaging and an order of magnitude or more less than reported doses for conventional CT. Dentomaxillofacial Radiology (2006) 35, 219-226. doi: 10.1259/dmfr/14340323
\end{abstract}

Keywords: radiation dosimetry; phantoms, imaging; risk assessment; tomography, X-ray computed

\section{Introduction}

Cone beam computed tomography (CBCT) provides a lower dose, lower cost alternative to conventional CT that promises to revolutionize the practice of oral and maxillofacial radiology. CBCT has been used in an expanding number of applications involving the disciplines of endodontics, ${ }^{1}$ oral surgery, ${ }^{2,3}$ oral medicine, ${ }^{4,5}$ periodontology, ${ }^{6}$ restorative dentistry ${ }^{7}$ and orthodontics. ${ }^{8}$ With the introduction of large (nominally $12^{\prime \prime}$ ) imaging fields, there has been a surge of interest in the use of CBCT as a

*Correspondence to: John B Ludlow, 120 Dental Office Building, UNC School of Dentistry, Chapel Hill, NC 27599-7450, USA; E-mail: jbl@email.unc.edu Received 7 November 2005; revised 15 December 2005; accepted 20 December 2005 substitute for conventional panoramic and cephalometric images for orthodontic treatment planning.

Although doses from CBCT are relatively low, patient dose remains a concern in dental diagnostic imaging. ${ }^{9}$ The possibility of a pituitary or thyroid link in the risk of low birth weight infants due to maternal exposures to low levels of dental X-ray is a recent example of a continuing scrutiny of potential radiation hazards from diagnostic imaging. ${ }^{10}$ Increasing use of CBCT examinations means that more patients are being subjected to the doses imparted from this procedure. To the extent that CBCT is used as a substitute for medical CT, patients will benefit from dose reduction. However, in the case where CBCT is substituted for lower dose conventional imaging alternatives, an increase in dose detriment is imparted to the patient. In the instance of the 
child orthodontic patient this is of particular concern because children are assumed to carry any radiation burden for a longer period of time than adults and because developing organs are more sensitive to radiation effects.

It is important for Oral and Maxillofacial Radiologists to know and communicate the dose and associated risk of specific examinations to their patients and referring practitioners. It is critical for healthcare providers to weigh the potential benefit of diagnostic information against the expense and risk of the imaging procedure. While early reports suggested that CBCT examination doses were equivalent to a few panoramic exposures, these reports were based on the unit of one vendor and a $9 "$ field of view (FOV). ${ }^{11,12}$ The current study provides comparative measurements of effective dose for three commercially available large $\left(12^{\prime \prime}\right)$ FOV CBCT units. Thermoluminescent dosemeters (TLDs) positioned throughout the layers of the head and neck of a tissue-equivalent RANDO phantom were used to record doses from full field and, when available, smaller field examinations. Average tissue-absorbed dose, radiation weighted dose and effective dose were calculated. The radiation weighted dose, formerly know by the terms dose equivalent and equivalent dose was calculated following both International Commission on Radiological Protection (ICRP) 1990 tissue weights ${ }^{13}$ and proposed 2005 tissue weights. ${ }^{14}$ Effective doses from these calculations were used in relative comparisons with conventional panoramic images and annual per capita background dose.

\section{Materials and methods}

TLD chips were used to record the distribution of the absorbed radiation dose at selected locations in the head and neck region of a small adult skull and tissue-equivalent phantom (RANDO - radiation analogue dosimetry system; Nuclear Associates, Hicksville, NY) (Figure 1).

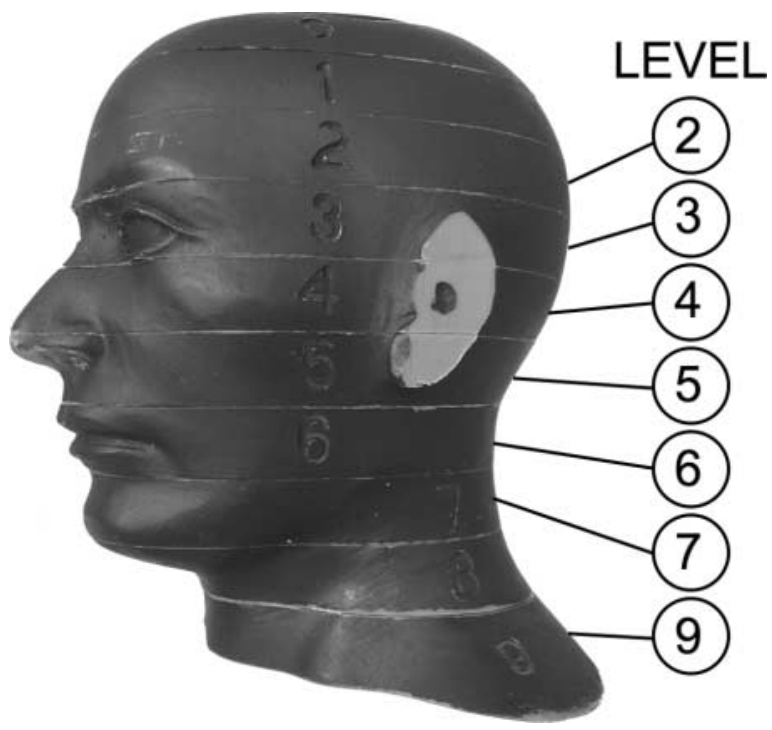

Figure 1 Adult skull and tissue-equivalent phantom (RANDO). Levels correspond to thermoluminescent dosemeter sites identified in Table 1
The 24 phantom sites measured in this study are listed in Table 1 . These locations reflect critical organs known to be sensitive to radiation, along with the eyes and the pituitary gland, which are sites of traditional and topical interest, respectively, to dental imaging. An unexposed dosemeter was also included for environmental calibration of each technique run. Pre-calibrated $3 \mathrm{~mm} \times 3 \mathrm{~mm} \times 1 \mathrm{~mm}$ TLD 100 lithium fluoride chips were supplied and analysed by Landauer Inc. (Landauer, Glenwood, IL). Landauer calibrated each dosemeter by exposing it to a known quantity of radiation from a Cs-137 source. Dosemeters were analysed using an automatic hot gas reader and the raw data were recorded. Individual TLD chip sensitivity was obtained and applied as a correction factor to subsequent exposure and reading of each TLD. The standard deviation of calibrated readings from the supplied TLD 100 chips is stated to be less than $\pm 5 \%$.

The CBCT units selected for this study were the $\mathrm{CB}$ Mercuray (Hitachi Medical Systems America, Twinsburg, $\mathrm{OH})$, the NewTom 3G (QR, Verona, Italy) and the i-CAT (Imaging Sciences International, Hatfield, PA). These units were chosen for the large $12^{\prime \prime}$ field of view (FOV) or beam diameter produced at the surface of the image receptor. This large FOV permits simultaneous imaging of the complete base of the skull as well as maxillofacial anatomy extending from the frontal process to the base of the chin. This anatomic region is utilized in craniometric calculations for orthodontic diagnosis and treatment planning. Full 12" FOV examinations were conducted on the RANDO phantom with each CBCT unit. Additional $9^{\prime \prime}$ and 6" FOV examinations were conducted with the $\mathrm{CB}$ Mercuray and $9^{\prime \prime}$ FOV with the i-CAT. At the time this research was conducted the NewTom $3 \mathrm{G}$ unit did not have a smaller FOV imaging option. This unit currently has a $9^{\prime \prime}$ and $6^{\prime \prime} \mathrm{FOV}$ as alternatives to the $12^{\prime \prime}$ FOV. Both of these units currently have these as available options. The phantom was orientated in each unit such that the phantom's occlusal plane was parallel to the scan plane. The midsagittal plane was centred in the image field and

Table 1 Locations of thermoluminescent dosemeter (TLD) chips in RANDO phantom

\begin{tabular}{lll}
\hline Organ & Location & Phantom level \\
\hline Bone marrow & Calvarium anterior & 2 \\
& Calvarium left & 2 \\
& Calvarium posterior & 2 \\
& Centre cervical spine & 6 \\
& Right/left mandible body & 7 \\
\multirow{3}{*}{ Brain } & Right/left ramus & 6 \\
\multirow{5}{*}{ Eyes } & Mid brain & 2 \\
\multirow{3}{*}{ Salivary glands } & Pituitary fossa & 3 \\
& Right/left orbit & 4 \\
\multirow{2}{*}{ Thyroid } & Right/left lens of eye & 4 \\
& Right/left parotid & 6 \\
Skin & Right/left submandibular gland & 7 \\
\multirow{2}{*}{ Oesophagus } & Sublingual gland & 7 \\
& Thyroid surface & 9 \\
& Midline thyroid & 9 \\
& Right cheek & 5 \\
& Left back of neck & 9 \\
\hline
\end{tabular}


the soft tissue contours of the chin and nose were captured at the margins of the field. Phantom levels 2-8 were included in the full FOV examinations produced by each unit. Midsagittal reconstructions resulting from these examinations can be seen in Figure 2.

$\mathrm{X}$-ray parameters of $\mathrm{kV}$ and $\mathrm{mA}$ are automatically determined from scout views by the NewTom $3 \mathrm{G}$. Depending on the size of the patient and the extent of beam attenuation a change in exposure of up to $40 \%$ is possible. The exposure settings for the i-CAT are fixed regardless of patient size. Technique factors of $\mathrm{mA}$ and $\mathrm{kV}$ are operator adjustable for the CB Mercuray. Technique factors of $120 \mathrm{kV}$ and $15 \mathrm{~mA}$ were used in initial examinations of the $\mathrm{CB}$ Mercuray. Using these factors the CB Mercuray examination was repeated after 3 months to determine examination reproducibility. Additional CB Mercuray imaging at $100 \mathrm{kV}$ and $10 \mathrm{~mA}$ was performed when it was found that these settings produced subjectively equivalent image quality for the phantom. Technical factors for each unit can be seen in Table 2. Owing to the relatively small amounts of radiation required for a single examination in comparison with the exposure latitude of the TLDs, multiple exposures for each radiographic technique were utilized to provide a reliable measure of radiation in the dosemeters. Three exposures were made without changing the position of the phantom for each CBCT examination variation.

Doses from TLDs at different positions within a tissue or organ were averaged to express the average tissueabsorbed dose in micrograys ( $\mu \mathrm{Gy}$ ). The products of these values and the percentage of a tissue or organ irradiated in a full FOV exam (Table 3) were used to calculate the radiation weighted dose $\left(H_{\mathrm{T}}\right)$ in microsieverts $(\mu \mathrm{Sv}){ }^{13}$

For bone marrow, the radiation weighted dose to the whole-body bone marrow is calculated using the summation of the individual radiation weighted dose to
Table 2 Technical factors for standard full field of view (FOV) exposure of RANDO phantom

\begin{tabular}{lccc}
\hline & CB Mercuray & i-CAT & NewTom $3 G$ \\
\hline $\mathrm{kV}$ & 100 & 120 & 110 \\
$\mathrm{~mA}$ & 10.0 & 5.7 & 1.5 \\
Total exposure time (s) & 10.0 & $6.6^{*}$ & 5.4 \\
Basis images & 288 & $300^{*}$ & 360 \\
Exposure time per image & 0.035 & 0.011 & 0.015 \\
Exposure arc subtended per image & $1.25^{\circ}$ & $0.20^{\circ}$ & $0.15^{\circ}$ \\
$\mathrm{mAs}$ & 100.0 & 37.3 & 8.1 \\
\hline
\end{tabular}

*The i-CAT uses 2 scans (lower face, upper face) and interlaces the scans to produce a full $12^{\prime \prime}$ FOV CT volume. Smaller FOVs are produced with a single scan

the calvarium, the mandible and the cervical spine. The determination of these radiation weighted doses is based on the distribution of active bone marrow throughout the adult body: the mandible contains $1.3 \%$, the calvaria contains $11.8 \%$ and the cervical spine contains $3.4 \% .^{15}$ Following the technique of Underhill et al, three locations within the calvarium were averaged to determine calvarial dose. ${ }^{16}$

The proportion of skin surface area in the head and neck region directly exposed by each technique was estimated as $5 \%$ of the total body to calculate radiation weighted dose to the skin following the procedure used in a previous study. ${ }^{11}$ Similarly, muscle, adipose, connective tissue and lymphatic nodes exposures were estimated to represent $5 \%$ of the total body complement for these tissues. The proportion of the oesophageal tract that was exposed was conservatively set at $10 \%$.

Effective dose $(E)$ is a widely used calculation that permits comparison of the detriment of different exposures to ionizing radiation to an equivalent detriment produced by a full body dose of radiation. $E$, expressed in $\mu \mathrm{Sv}$, was calculated using the equation: $E=\Sigma w_{\mathrm{T}} \times H_{\mathrm{T}}$, where $E$ is the product of the tissue weighting factor $\left(w_{\mathrm{T}}\right)$, which represents

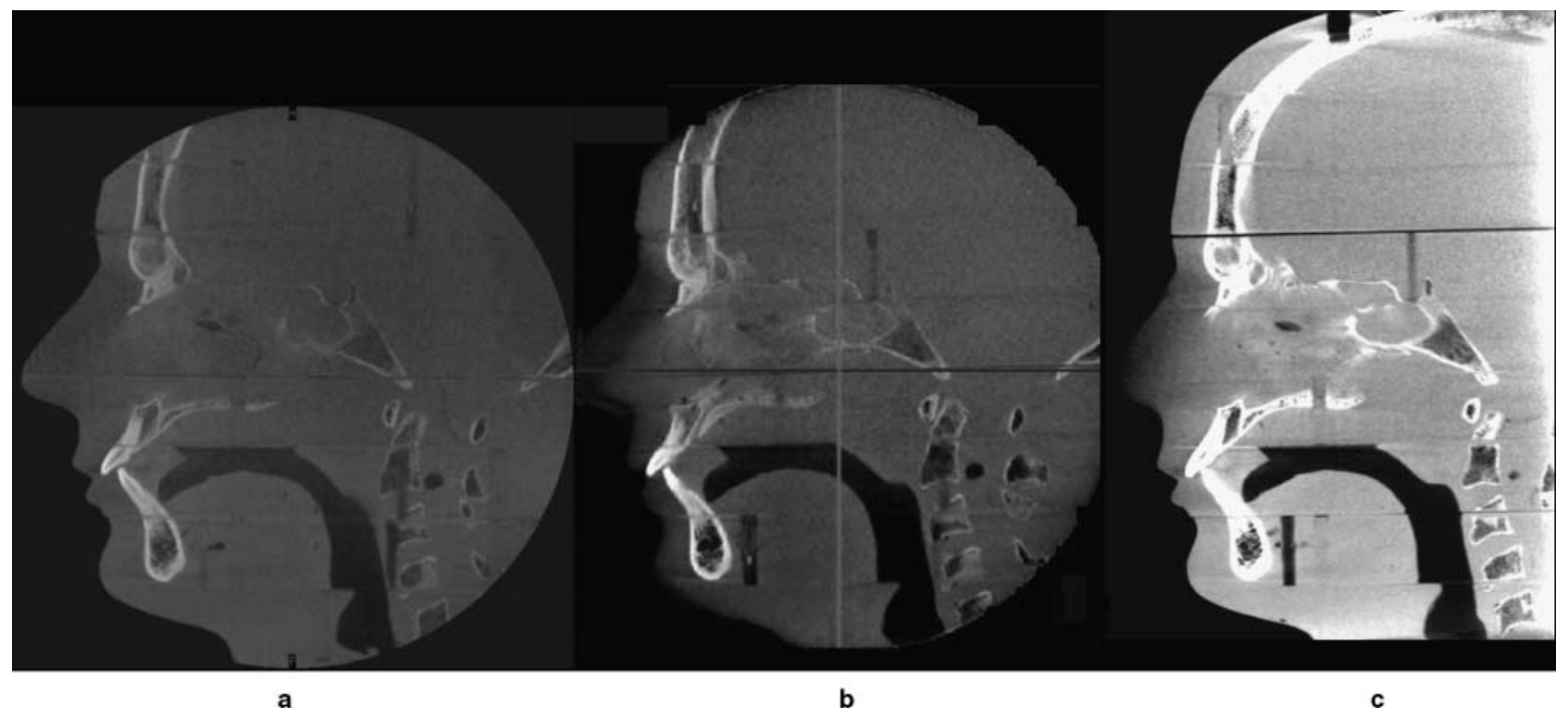

Figure 2 Lateral views demonstrating equivalent phantom positioning for $12^{\prime \prime}$ field of view (FOV) examination: (A) CB Mercuray midsagittal reconstruction, (B) NewTom 3G midsagittal reconstruction, (C) i-CAT midsagittal reconstruction 
Table 3 Estimated percentage of tissue irradiated and thermoluminescent dosemeters (TLDs) used to calculate mean absorbed dose to a tissue or organ

\begin{tabular}{|c|c|c|}
\hline & Fraction irradiated (\%) & $\begin{array}{l}\text { TLD ID } \\
\text { (see Table 5) }\end{array}$ \\
\hline Bone marrow & 16.5 & \\
\hline Mandible & 1.3 & $13,14,17,18$ \\
\hline Calvaria & 11.8 & 1,3 \\
\hline Cervical spine & 3.4 & 15 \\
\hline Thyroid & 100 & 22,23 \\
\hline Oesophagus & 10 & 24 \\
\hline Skin & 5 & $8,9,10,16$ \\
\hline Bone surface $^{a}$ & 16.5 & \\
\hline Mandible & 1.3 & $13,14,17,18$ \\
\hline Calvaria & 11.8 & 1,3 \\
\hline Cervical spine & 3.4 & 15 \\
\hline Salivary glands & 100 & \\
\hline Parotid & 100 & 11,12 \\
\hline Submandibular & 100 & 19,20 \\
\hline Sub-lingual & 100 & 21 \\
\hline Brain $^{b}$ & 100 & 4,5 \\
\hline \multicolumn{3}{|l|}{ Remainder } \\
\hline Brain $^{c}$ & 100 & 4,5 \\
\hline Adipose $^{b}$ & 5 & $11,12,19-21$ \\
\hline Connective tissue ${ }^{b}$ & 5 & $11,12,19-21$ \\
\hline Lymphatic nodes ${ }^{b}$ & 5 & $11,12,19-21$ \\
\hline Muscle $^{a b}$ & 5 & $6,7,15,21,24$ \\
\hline Extrathoracic airway $^{b}$ & 100 & $11,12,19-21$ \\
\hline Pituitary & 100 & 5 \\
\hline Eyes & 100 & $6,7,8,9$ \\
\hline
\end{tabular}

${ }^{a}$ Bone surface dose $=$ bone marrow dose $\times 4.64 ;{ }^{b}$ ICRP $2005^{14} ;{ }^{c}$ ICRP $1990^{13}$

the relative contribution of that organ or tissue to the overall risk, and the radiation weighted dose $H_{\mathrm{T}}{ }^{13}$ The whole-body risk is found by the summation of the radiation weighted doses to all tissues or organs exposed. Both current 1990 ICRP tissue weights and proposed 2005 weights found in Table 4 were used to calculate effective dose. ${ }^{13,14}$

The 1990 weighting factors have been assigned to 12 organs or tissues and a group of remainder organs for purposes of calculating total $E$. Of the individually weighted tissues or organs only bone marrow, oesophagus,

Table 4 Tissue weighting factors for calculation of effective dose ICRP $1990^{13}$ and 2005 draft recommendations ${ }^{14}$

\begin{tabular}{lll}
\hline Tissue & $1990 w_{T}$ & $2005 w_{T}$ \\
\hline Bone marrow & 0.12 & 0.12 \\
Breast & 0.05 & 0.12 \\
Colon & 0.12 & 0.12 \\
Lung & 0.12 & 0.12 \\
Stomach & 0.12 & 0.12 \\
Bladder & 0.05 & 0.05 \\
Oesophagus & 0.05 & 0.05 \\
Gonads & 0.20 & 0.05 \\
Liver & 0.05 & 0.05 \\
Thyroid & 0.05 & 0.05 \\
Bone surface & 0.01 & 0.01 \\
Brain & remainder & 0.01 \\
Kidneys & remainder & 0.01 \\
Salivary glands & - & 0.01 \\
Skin & 0.01 & 0.01 \\
Remainder tissues & $0.05^{a}$ & $0.10^{b}$ \\
\hline
\end{tabular}

${ }^{a}$ Adrenals, brain, upper large intestine, small intestine, kidney, muscle, pancreas, spleen, thymus, uterus; ${ }^{b}$ Adipose tissue, adrenals, connective tissue, extrathoracic airways, gall bladder, heart wall, lymphatic nodes, muscle, pancreas, prostate, SI wall, spleen, thymus, and uterus/cervix thyroid, bone surface and skin doses were included in this study. Of the ten organs making up the remainder category, only brain and muscle were included. The other individual or remainder organs are not directly exposed in the protocols used in this study. While an assumption of no dose may underestimate actual exposure to these organs, the impact on total $E$ is negligible. Proposed tissue weights for 2005 increase the number of independently weighted tissues by 2 and expand the number of remainder tissues to 14. Remainder tissues directly exposed in the full FOV CBCT exam include adipose, connective tissue, lymphatic nodes, muscle and extrathoracic airway.

\section{Results}

Average dosemeter readings after three CBCT exposure cycles ranged from $2.5 \mathrm{mGy}$ for the full FOV NewTom $3 \mathrm{G}$ examination to $32.7 \mathrm{mGy}$ for the full FOV CB Mercuray examination made at $120 \mathrm{kV}$ and $15 \mathrm{~mA}$. These doses are well above the minimum detection threshold of $0.3 \mathrm{mGy}$ for the TLD 100 chips. Table 5 provides dosemeter values for the repeated full FOV exam with the CB Mercuray using $120 \mathrm{kV}$ and $15 \mathrm{~mA}$ exposure factors. Overall reproducibility, within the limits of dosemeter error, is indicated by an average dose difference of $2.5 \%$ between repeated examinations. Radiation weighted dose $H_{\mathrm{T}}$ and effective dose $E$ calculations for the standard full FOV exams for each of the CBCT units are seen in Table 6. $E_{\text {(ICRP1990) }}$ and $E_{\text {(ICRP2005 draft })}$ for the NewTom $3 \mathrm{G}$ were 44.5, 58.9; for the i-CAT 134.8, 193.4; and for the CB Mercuray 476.6, 557.6. As a relative multiple of the NewTom $3 \mathrm{G}$ full FOV dose, the i-CAT examination resulted in 1.5 times more dose while the $\mathrm{CB}$ Mercuray required 11 times more dose as calculated using $E_{\text {(ICRP1990) }}$ values. Dose multiples using $E_{\text {(ICRP2005 draft) }}$ values were 3.3 for the i-CAT and 9.5 for the CB Mercuray. Comparisons of $E_{\text {(ICRP1990) }}$ and $E_{\text {(ICRP2005 draft) }}$ for different size FOVs as a multiple of panoramic examinations and as a percentage of annual per capita background dose from all sources are seen in Table 7.

\section{Discussion}

Comparison of multiple CBCT units using the same human dosimetry phantom has not previously been done. Utilizing the same phantom in equivalent full FOV examinations with currently available $9^{\prime \prime}$ and $12^{\prime \prime}$ FOV units permits a relative comparison of their dosimetric performance. While some aspects of dosemeter site selection and handling of fractionally irradiated tissues have been addressed by prior studies, newly included adipose, connective tissue, lymphatic nodes, muscle and extrathoracic airway tissues in the proposed ICRP 2005 tissue weighting scheme have not previously been addressed in the dental literature. This study took a simplistic and arbitrary approach in placing the body proportion of these tissues exposed in a full FOV CBCT exam at 5\%. As the distribution of these tissues in the body is non-uniform, the $5 \%$ figure may overestimate or underestimate the actual proportion of each tissue in the X-ray field. It is expected 
Table 5 Dosimetry reproducibility: Mercuray CB Full field of view (FOV) - $120 \mathrm{kV}, 15 \mathrm{~mA}$

\begin{tabular}{|c|c|c|c|c|}
\hline Phantom location & $T L D I D$ & Scan $1(m G y)$ & Scan $2(m G y)$ & Percent variation 2 from 1 \\
\hline Calvarium anterior (2) & 1 & 8.97 & 10.20 & $13 \%$ \\
\hline Calvarium left (2) & 2 & 9.93 & 10.70 & $7 \%$ \\
\hline Calvarium posterior (2) & 3 & 6.87 & 7.43 & $8 \%$ \\
\hline Mid brain (2) & 4 & 9.07 & 9.33 & $3 \%$ \\
\hline Pituitary (3) & 5 & 9.30 & 9.40 & $1 \%$ \\
\hline Right orbit (4) & 6 & 10.03 & 10.10 & $1 \%$ \\
\hline Left orbit (4) & 7 & 9.93 & 10.33 & $4 \%$ \\
\hline Right lens of eye (3) & 8 & 16.37 & 16.70 & $2 \%$ \\
\hline Left lens of eye (3) & 9 & 15.70 & 16.37 & $4 \%$ \\
\hline Right cheek (5) & 10 & 15.43 & 15.70 & $2 \%$ \\
\hline Right parotid (6) & 11 & 14.40 & 14.67 & $2 \%$ \\
\hline Left parotid (6) & 12 & 13.30 & 14.23 & $7 \%$ \\
\hline Right ramus (6) & 13 & 9.47 & 10.20 & $7 \%$ \\
\hline Left ramus (6) & 14 & 9.73 & 10.60 & $9 \%$ \\
\hline Centre cervical spine (6) & 15 & 9.60 & 10.83 & $12 \%$ \\
\hline Left back of neck (7) & 16 & 12.53 & 17.33 & $32 \%$ \\
\hline Right mandible body (7) & 17 & 9.37 & 10.27 & $9 \%$ \\
\hline Left mandible body (7) & 18 & 9.57 & 11.00 & $14 \%$ \\
\hline Right submandibular gland (7) & 19 & 10.70 & 11.53 & $7 \%$ \\
\hline Left submandibular gland (7) & 20 & 11.37 & 11.53 & $1 \%$ \\
\hline Centre sublingual gland (7) & 21 & 9.83 & 10.30 & $5 \%$ \\
\hline Midline thyroid (9) & 22 & 11.00 & 11.40 & $4 \%$ \\
\hline Thyroid surface - left (9) & 23 & 12.93 & 4.83 & $-91 \%$ \\
\hline Oesophagus (9) & 24 & 6.30 & 6.13 & $-3 \%$ \\
\hline Average TLD dose & & 10.90 & 11.30 & $2.50 \%$ \\
\hline
\end{tabular}

that future studies may refine the numeric used in calculating proportions of tissues in the head and neck area as well as the best location for dosemeter placement for measuring exposure. Despite the uncertainty of effect of these definitional and experimental uncertainties on an absolute measure of effective dose, the relative comparison of dose between units is valid.

Reproducibility of the dosimetric technique utilized in this study was affirmed by the $2.5 \%$ overall variation between repeated CB Mercuray examinations. However, there were significant deviations for specific dosemeter locations. This was especially apparent in dosemeters placed on the skin surface and dosemeters placed near the

Table 6 Radiation weighted dose ${ }^{\S} H_{\mathrm{T}}(\mu \mathrm{Sv})$ and effective dose $E(\mu \mathrm{Sv})$ for full $\left(\approx 12^{\prime \prime}\right)$ field of view (FOV) exposures for 3 cone beam computed tomography (CBCT) units

\begin{tabular}{lccc}
\hline Organ or tissue & NewTom $3 G$ & i-CAT & CB Mercuray \\
\hline Bone marrow & 125 & 418 & 692 \\
Thyroid & 333 & 767 & 6333 \\
Oesophagus & 57 & 123 & 393 \\
Skin & 62 & 187 & 389 \\
Bone surface & 581 & 1941 & 3211 \\
Salivary glands & 956 & 3522 & 5467 \\
Brain* & 700 & 3583 & 3967 \\
Remainder & & & \\
Brain $^{\dagger}$ & 700 & 3583 & 3967 \\
Adipose* & 48 & 176 & 273 \\
Connective tissue* $^{*}$ & 48 & 176 & 273 \\
Lymphatic nodes* $^{*}$ & 48 & 176 & 273 \\
Mutrathoracic airway* $^{*}$ & 760 & 3733 & 4813 \\
Pituitary & 48 & 176 & 273 \\
Eyes $^{* \dagger}$ & 733 & 4233 & 4000 \\
Effective Dose $^{\dagger}$ & 1017 & 5008 & 6208 \\
Effective Dose* $^{*}$ & 44.7 & 134.8 & 476.6 \\
\hline
\end{tabular}

"ICRP 2005 draft $^{14}$; ${ }^{\dagger}$ ICRP $1990^{13}$; ${ }^{8}$ Formerly know as Equivalent dose caudal and cranial extremes of the X-ray field. In particular the thyroid surface detector exposure varied $91 \%$ between repeated examinations. The next largest variation occurred with the dosemeter placed at the back of the neck. It was felt that the small anterior-posterior position differences between the two exposures coupled with a dosemeter location at the posterior periphery of laterally directed projections may have played a role in the reading difference of $32 \%$. Internally positioned TLDs are constrained by pre-drilled holes that snugly accommodate the TLD and its protective envelope. Surface TLDs were taped in place. The actual position of the TLD in its envelope could have varied by as much as a centimetre. Just as the position of dosemeters at the edge of the X-ray field can impact recorded exposure, position of the patient can also have a significant impact on dose to critical organs. As a critical peripheral organ, the position of the thyroid can be manipulated by a number of strategies including upward tipping of the chin and use of smaller FOVs to reduce dose.

While full FOV doses from the dental CBCT units in this study were $2-23 \%$ of the dose of comparable conventional CT examinations reported in the literature, ${ }^{17}$ they were also several to hundreds of times greater than single panoramic image exposures. It is hoped that a CBCT examination would not be substituted for a panoramic examination if a panoramic image alone would be adequate. In the case of orthodontic diagnosis, substitution of the CBCT for panoramic and lateral and posterior-anterior (PA) cephalometric images may be contemplated. Dose calculations using ICRP 1990 tissue weights are $6.2 \mu \mathrm{Sv}$ for a direct digital panoramic image ${ }^{11}$ and $3.4 \mu \mathrm{Sv}$ for 2 cephalometric images. ${ }^{18}$ If the calculation of $E$ is modified to include salivary glands then the panoramic and cephalometric doses 


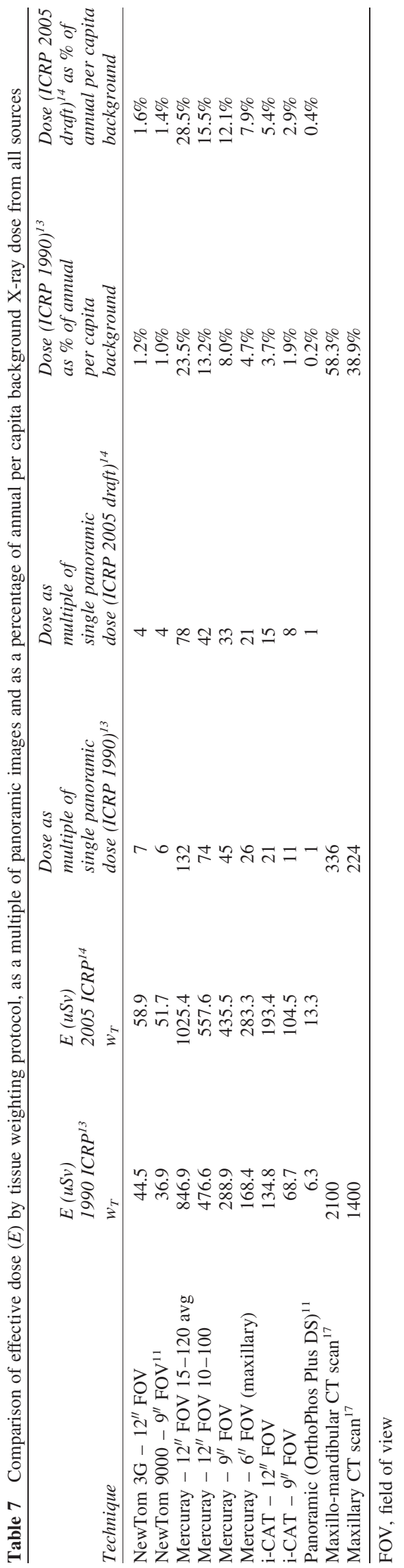

increase to $22 \mu \mathrm{Sv}$ and $6.8 \mu \mathrm{Sv}$, respectively. The full FOV NewTom $3 \mathrm{G}$ examination is between 2 ( $\left.E_{\text {(ICRP2005 draft })}\right)$ and $4.5\left(E_{\text {(ICRP1990) }}\right)$ times these conventional exposures.

In comparison with conventional plain radiography, the potential for obtaining substantial additional information from a CBCT volume is tremendous. Even in obtaining the "same" information as that derived from conventional views, the clinician is freed from the constraints of cephalometric orientation, the problems of geometric distortion, and many of the challenges of separating cephalometric landmarks from structure noise. In addition new ways of observing the data (maximum intensity projections, multiplanar reconstructions, rotations in 3D) may provide diagnostic insights that were not here-to-fore possible. While additional diagnostic information may be available in the CBCT volume, the question that must be asked is "will this additional information contribute to the diagnosis, and will it have a positive impact on the patient's treatment?"

If the clinician does not obtain significantly better diagnostic information for the patient where CBCT is substituted for a conventional orthodontic imaging series, how concerned should he/she be about the additional dose? On one hand the NewTom 3G doses are 4-6 days of equivalent per capita background dose. On the other hand CB Mercuray doses are 48-56 days of background dose. To provide further prospective, the $150 \mu \mathrm{Sv}$ associated with the most common full mouth radiographic examination (FMX) (D speed film, round collimation) utilizes about 3 times the dose of the NewTom 3G exam and about one third of the dose of the CB Mercuray exam. ${ }^{19}$ However, it should be noted that current NCRP guidelines for dentistry recommend rectangular collimation and $\mathrm{E}$ or F speed X-ray detectors, which would reduce the FMX dose by a factor of 6 or more. ${ }^{20}$

It is noteworthy that smaller FOV examinations are associated with significant dose reductions. In the case of the CB Mercuray, the $9^{\prime \prime}$ FOV examination was produced with $60 \%\left(E_{\text {(ICRP1990) }}\right)$ to $75 \%\left(E_{\text {(ICRP2005 draft })}\right)$ of the dose of the standard $12^{\prime \prime}$ FOV exam, while the $6^{\prime \prime}$ FOV exam centred on the maxilla required only a third $\left(E_{\text {(ICRP1990) }}\right)$ to half $\left(E_{\text {(ICRP2005 draft }}\right)$ of the full FOV dose. To the extent that a smaller FOV can be used to supply the needed diagnostic information this approach should be used. A common example of this would be the utilization of CBCT for implant treatment planning. A single jaw is readily imaged by a $6^{\prime \prime}$ FOV and both jaws are completely visualized by a $9^{\prime \prime}$ FOV.

It is not enough to simply compare doses of alternate imaging modalities. Issues of diagnostic quality cannot be divorced from issues of dose. This study did not address image quality. Exposure factors set by or recommended by the manufacturer were used for standard exposures. In the case of the CB Mercuray, which has user adjustable technique factors, the maximum technique combination was used to determine an upper limit of exposure from this unit. In comparing subjectively the quality of images produced with $120 \mathrm{kV}$ and $15 \mathrm{~mA}$ with those produced by $100 \mathrm{kV}$ and $10 \mathrm{~mA}$, little difference could be seen. The lower dose images were slightly noisier. Comparing standard CB Mercuray images with i-CAT and NewTom 
$3 \mathrm{G}$ images produced the same impression. While the $\mathrm{CB}$ Mercuray images were less noisy, it was subjectively similar in quality to the other units. Objective studies of the effect of CBCT image quality on diagnostic performance need to be conducted before definitive conclusions can be made about the importance of the subjective differences in image quality resulting from reduced exposure.

Examining the technical characteristics of the three CBCT units in Table 2 shows that the principal factor accounting for differences in dose in full FOV examinations is mAs. While the NewTom 3G scans patients over a $36 \mathrm{~s}$ period, exposure takes place for only $5.4 \mathrm{~s}$. Similarly, the i-CAT 9 "FOV scans over a $20 \mathrm{~s}$ interval but the X-ray tube is activated for only $3.3 \mathrm{~s}$. The i-CAT implementation of a nominal 12 "FOV is termed an "extended field of view" and involves two $9^{\prime \prime}$ FOV scans, made sequentially and stitched together to form a larger volume. Use of this double scan technique preserves the resolution of the $9^{\prime \prime}$ scan, but requires almost twice the exposure time and twice the exposure. The CB Mercuray scans for about $11 \mathrm{~s}$ while the tube produces X-rays for $10.0 \mathrm{~s}$. While the CB Mercuray exposes continuously, the NewTom $3 \mathrm{G}$ and i-CAT pulse radiation. This results in a more efficient use of dose because the detector is only being exposed while it is recording photons. During periods when the detector is transferring its image signal to the computer, radiation is turned off. Another factor of potential importance is the sensitivity of the detector. The i-CAT utilizes an amorphous silicon flat panel detector while the NewTom 3G and the CB Mercuray incorporate an image intensifier and charge coupled device (CCD) detector. Although differences in the sensitivity of the X-ray detectors could play a role in dose efficiency it is unknown what role, if any, this factor played in the differences in dosimetry observed in this study.

The approach taken by each CBCT unit in setting exposure factors is quite different. The simplest method is used by the i-CAT where $\mathrm{kV}, \mathrm{mA}$, and exposure time are established by the manufacturer and do not vary from patient to patient. This technique requires that the dose be sufficient to accommodate both large and small patients. For smaller patients, such as children, this may produce higher doses than required for diagnostic quality. The NewTom 3G exposure is also fixed by the manufacturer. However, a dynamic process is used where the quantity of radiation required for a particular patient is determined from PA and lateral scout views and the $\mathrm{mA}$ of the unit is adjusted accordingly during the exposure. It is likely that the small adult phantom used in this study resulted in a lower dose with the NewTom $3 \mathrm{G}$ than would be seen with a larger phantom or patient. With the CB Mercuray, the operator sets factors of $\mathrm{mA}$ and $\mathrm{kV}$. CBCT images provide little feedback to indicate excessive exposure. After window and levelling, overexposed images look good and if anything are less noisy than properly exposed volumes. Among inexperienced operators there may be a tendency to maximize $\mathrm{kV}$ and $\mathrm{mA}$ settings. Images always appear adequately exposed and noise is minimized by this approach. With the majority of CBCT installations currently taking place in orthodontic offices, the risk of overexposure from the user adjustment technique is great.
The principal reason for revising tissue weighting factors in the proposed 2005 ICRP draft document is the availability of cancer incidence data that was not available when the 1990 guidelines were published. ICRP 1990 cancer risks were computed based on mortality data. Incidence data provide a more complete description of cancer burden than do mortality data, particularly for cancers that have a high survival rate. Much of the cancer incidence data comes from the Life Span Study (LSS) of Japanese atomic bomb survivors which has been updated with follow-up through 1998, and has been corrected using DS86 bomb dosimetry. Weighted tissues and organs were selected in the 2005 revision because of sufficient epidemiological information on the tumorigenic effects of radiation to make judgments necessary for estimating cancer risks. Changes include an increase in the detriment of thyroid cancer to 0.05 due to the concentration of cancer risk in childhood. Cancer risk in salivary glands, brain and kidney were judged to be greater than that of other tissues in the remainder fraction and each is ascribed a $w_{\mathrm{T}}$ of 0.01 . A $w_{\mathrm{T}}$ value for the remainder tissues of 0.1 , distributed equally amongst 14 named tissues, provides a weight of approximately 0.007 each, which is lower than the $w_{\mathrm{T}}$ for the lowest of the named tissues. ${ }^{14}$

The general increase in $E_{\text {(ICRP2005 draft) }}$ from $E_{\text {(ICRP1990) }}$ calculations seen in this study is largely due to the inclusion of salivary glands as a weighted tissue in $E_{\text {(ICRP2005 draft) }}$ calculations. Moving brain from the remainder group with provision of an independent weight of 0.01 contributes to a lesser extent to the increase. While calculated $E_{\text {(ICRP2005 draft) }}$ doses indicate a greater detriment from diagnostic imaging relative to per capita background dose, detriment as a function of multiples of panoramic images has actually decreased (Table 7). The reason for this is that the locations of the posterior rotational centres for panoramic scanning motions are located near the parotid and submandibular glands while the anterior rotational centre is located near the sublingual gland. The salivary glands receive almost continuous direct exposure during a panoramic examination and thus absorb a disproportionately larger dose of radiation. CBCT examinations result in a much more uniform exposure of maxillofacial tissues.

The potential of significantly improved diagnostic protocols and the long-term benefit of improved patient care easily justify additional increments of small risk. The results of this study do not provide a uniform picture about the risks involved with current full FOV CBCT examinations. Much additional work remains to be done. The benefits of specific examination protocols need to be validated with controlled prospective studies. In the absence of such validation, practitioners need to apply the skill, care, and judgment that sets them apart as healthcare professionals in determining when and what examination is indicated. When possible $\mathrm{mA}$ and $\mathrm{kV}$ should be adjusted to minimize dose. The utilized dose should be tailored to the diagnostic task with thoughtful choice of FOV, and careful orientation of anatomy within the field of view. CBCT technology continues to evolve and new or improved units will appear in the marketplace in the future. Both image quality and dosimetry 
information is needed on these units so that buyers can make informed choices about the appropriateness of a particular unit for their diagnostic needs. CBCT dose varies substantially depending on the device, FOV and selected technique factors. Full FOV examination i-CAT doses were $3\left(E_{(\mathrm{ICRP} 1990)}\right)$ to $3.3\left(E_{(\mathrm{ICRP} 2005}\right.$ draft $)$ times greater than NewTom $3 \mathrm{G}$ doses while Mercuray doses were $10.7\left(E_{(\mathrm{ICRP} 1990)}\right)$ to $9.5\left(E_{(\mathrm{ICRP} 2005 \text { draft })}\right)$ times greater. Effective dose detriment of currently available large FOV

\section{References}

1. Rigolone M, Pasqualini D, Bianchi L, Berutti E, Bianchi SD. Vestibular surgical access to the palatine root of the superior first molar: "low-dose cone-beam" CT analysis of the pathway and its anatomic variations. J Endod 2003; 29: 773-775.

2. Nakagawa Y, Kobayashi K, Ishii H, Mishima A, Ishii H, Asada K, et al. Preoperative application of limited cone beam computerized tomography as an assessment tool before minor oral surgery. Int $J$ Oral Maxillofac Surg 2002; 31: 322-326.

3. Hamada Y, Kondoh T, Noguchi K, Iino M, Isono H, Ishii H, et al. Application of limited cone beam computed tomography to clinical assessment of alveolar bone grafting: a preliminary report. Cleft Palate Craniofac J 2005; 42: 128-137.

4. Ogawa T, Enciso R, Memon A, Mah JK, Clark GT. Evaluation of 3D airway imaging of obstructive sleep apnea with cone-beam computed tomography. Stud Health Technol Inform 2005; 111: 365-368.

5. Tsiklakis K, Syriopoulos K, Stamatakis HC. Radiographic examination of the temporomandibular joint using cone beam computed tomography. Dentomaxillofac Radiol 2004; 33: 196-201.

6. Sato S, Arai Y, Shinoda K, Ito K. Clinical application of a new conebeam computerized tomography system to assess multiple twodimensional images for the preoperative treatment planning of maxillary implants: case reports. Quintessence Int 2004; 35: $525-528$.

7. Hatcher DC, Dial C, Mayorga C. Cone beam CT for pre-surgical assessment of implant sites. J Calif Dent Assoc 2003; 31: 825-833.

8. Maki K, Inou N, Takanishi A, Miller AJ. Computer-assisted simulations in orthodontic diagnosis and the application of a new cone beam X-ray computed tomography. Orthod Craniofac Res 2003; 6(Suppl. 1): $95-101$.

9. Farman AG. ALARA still applies. Oral Surg Oral Med Oral Pathol Oral Radiol Endod 2005; 100: 395-397.

10. Hujoel PP, Bollen AM, Noonan CJ, del Aguila MA. Antepartum dental radiography and infant low birth weight. JAMA 2004; 291: $1987-1993$
CPCT units is several to many times higher than conventional panoramic imaging and several to many times lower than reported doses for conventional CT.

\section{Acknowledgments}

Processing of dosemeters was funded in part by Hitachi Medical Systems America, Twinsburg, OH; Aperio Services, Sarasota, FL; and Imaging Sciences International, Hatfield, PA.

11. Ludlow JB, Davies-Ludlow LE, Brooks SL. Dosimetry of two extraoral direct digital imaging devices: NewTom cone beam CT and Orthophos Plus DS panoramic unit. Dentomaxillofac Radiol 2003. 32: $229-234$.

12. Mah JK, Danforth RA, Bumann A, Hatcher D. Radiation absorbed in maxillofacial imaging with a new dental computed tomography device. Oral Surg Oral Med Oral Pathol Oral Radiol Endod 2003; 96: $508-513$.

13. 1990 Recommendations of the International Commission on Radiological Protection, ICRP Publication 60. Ann ICRP 1991; 21: $1-201$.

14. Draft document "2005 Recommendations of the International Commission on Radiological Protection". ICRP http://www.icrp.org [Accessed 1 March 2006].

15. White SC, Rose TC. Absorbed bone marrow dose in certain denta radiographic techniques. J Am Dent Assoc 1979; 98: 553-558.

16. Underhill TE, Chilvarquer I, Kimura K, Langlais RP, McDavid WD, Preece JW, et al. Radiobiologic risk estimation from dental radiology, Part I, Absorbed doses to critical organs. Oral Surg Oral Med Oral Pathol 1988; 66: 111-120.

17. Ngan DC, Kharbanda OP, Geenty JP, Darendeliler MA. Comparison of radiation levels from computed tomography and conventional dental radiographs. Aust Orthod J 2003; 19: 67-75.

18. Gijbels F, Sanderink G, Wyatt J, Van Dam J, Nowak B, Jacobs R. Radiation doses of indirect and direct digital cephalometric radiography. Br Dent J 2004; 197: 149-152.

19. Avendanio B, Frederiksen NL, Benson BW, Sokolowski TW. Effective dose and risk assessment from detailed narrow beam radiography. Oral Surg Oral Med Oral Pathol Oral Radiol Endod 1996; 82: 713-719.

20. Brand JW, Gibbs SJ, Edwards M, Katz JO, Lurie AG, White SC Radiation Protection in Dentistry. NCRP 2003; Report No. 145. 